\title{
ON THREE-DIMENSIONAL CR YAMABE SOLITONS
}

\author{
HUAI-DONG $\mathrm{CAO}^{1},{ }^{*} \mathrm{SHU}-\mathrm{CHENG} \mathrm{CHANG}^{2}, \mathrm{AND}{ }^{*} \mathrm{CHIH}$-WEI CHEN ${ }^{3}$
}

\begin{abstract}
In this paper, we investigate the geometry and classification of threedimensional CR Yamabe solitons. In the compact case, we show that any 3-dimensional CR Yamabe soliton must have constant Tanaka-Webster scalar curvature; we also obtain a classification under the assumption that their potential functions are in the kernel of the CR Paneitz operator. In the complete case, we obtain a structure theorem on the diffeomorphism types of complete 3-dimensional pseudo-gradient CR Yamabe solitons (shrinking, or steady, or expanding) of vanishing torsion.
\end{abstract}

\section{INTRODUCTION}

Inspired by Perelman's work [P1, P2] on Hamilton's Ricci flow (cf. [H1]-[H5]), selfsimilar solutions, also known as (geometric) solitons, of various geometric flows have attracted a lot of attentions in recent years because of their close ties with singularity formations in the geometric flows. In particular, important progress has been made in the study of Ricci solitons, self-similar solutions to the mean curvature flow, as well as Yamabe solitons, etc.

For pseudohermitian manifolds, similar to the concept of Yamabe solitons, one can introduce the notion of CR Yamabe solitons and pseudo-gradient CR Yamabe solitons.

Definition 1.1. A pseudohermitian $(2 n+1)$-manifold $\left(M^{2 n+1}, J, \theta\right)$, with CR structure $J$ and compatible contact form $\theta$, is called a CR Yamabe soliton if there exist an infinitesimal contact diffeomorphism $X$ and a constant $\mu \in \mathbb{R}$ such that

$$
\left\{\begin{array}{l}
W \theta+\frac{1}{2} L_{X} \theta=\mu \theta \\
L_{X} J=0
\end{array}\right.
$$

where $W$ is the Tanaka-Webster scalar curvature of $\left(M^{2 n+1}, J, \theta\right)$ and $L_{X}$ denotes Lie derivative by $X$. It is called shrinking if $\mu>0$, steady if $\mu=0$, and expanding if $\mu<0$.

Remark 1.1. An infinitesimal contact diffeomorphism is a vector field $X$ on $M$ such that $L_{X} \theta=\lambda \theta$ for some function $\lambda$. It is known that for any infinitesimal contact diffeomorphism $X$, there always associates a function $f$ such that $X=J\left(\nabla_{b} f\right)+f \mathbf{T}$ and $L_{X} \theta=f_{0} \theta$, where $\nabla_{b}$ is the subgradient, and the subscript 0 denotes the differentiation along the Reeb vector field $\mathbf{T}$. Note that the first equation in (1.1) can then be expressed as $W+\frac{1}{2} f_{0}=\mu$, while the second equation $L_{X} J=0$ is corresponding to $f_{\alpha \alpha}+i A_{\alpha \alpha} f=0$ with torsion tensor $A=\left\{A_{\alpha \alpha}\right\}$. We refer the reader to Section 3 for more details.

As we shall see, CR Yamabe solitons also correspond to self-similar solutions to the CR Yamabe flow on a pseudohermitian $(2 n+1)$-manifold $\left(M^{2 n+1}, J, \stackrel{\circ}{\theta}\right)$ given by (cf.

2010 Mathematics Subject Classification. Primary 32V05; Secondary 53C12.

Key words and phrases. CR Harnack quantity, CR Yamabe soliton, CR Paneitz operator.

* Research supported in part by the NSC of Taiwan. 
CC]

$$
\left\{\begin{array}{l}
\frac{\partial}{\partial t} \theta(t)=-2 W(t) \theta(t), \\
\theta(0)=\stackrel{\theta}{ }
\end{array}\right.
$$

where $W(t)$ is the Tanaka-Webster scalar curvature with respect to the evolving contact form $\theta(t)$. Like the Riemannian Yamabe flow, which has been very well understood in the compact case, the compact CR Yamabe flow hopefully provides a canonical deformation from the given contact form $\dot{\theta}$ to a contact form $\theta$ of constant Tanaka-Webster scalar curvature. As noted in [CC], the CR Yamabe flow has a unique short time solution on any compact pseudohermitian $(2 n+1)$-manifold $\left(M^{2 n+1}, J, \stackrel{\circ}{\theta}\right)$. Moreover, on a compact pseudohermitian 3 -manifold $\left(M^{3} J, \stackrel{\circ}{\theta}\right)$, solutions to the normalized CR Yamabe flow

$$
\frac{\partial}{\partial t} \theta(t)=2(r(t)-W(t)) \theta(t) ; \quad r(t)=\int_{M} W(t) d \mu / \int_{M} d \mu
$$

exist for all time. This long time existence of the three-dimensional normalized CR Yamabe flow was established in [H6] and [SS]. However, unlike the Riemannian Yamabe flow, the problem of asymptotic convergence of solutions of the CR Yamabe flow is widely open even on closed pseudohermitian 3-manifolds. Here we propose the following

Conjecture 1.1. Let $\left(M^{3}, J, \stackrel{\circ}{\theta}\right)$ be a closed spherical CR 3-manifold with positive Tanaka-Webster scalar curvature and vanishing torsion. Then, as $t \rightarrow \infty$, the solution to the normalized CR Yamabe flow converges smoothly to a unique limit contact form of positive constant Tanaka-Webster scalar curvature and vanishing pseudohermitian torsion.

A related result was derived in a paper of the second named author with others CCW], where they proved the stability property for solutions of the normalized CR Yamabe flow near the standard CR 3-sphere $\left(\mathbf{S}^{3}, \widehat{J}, \widehat{\theta}\right)$ which is spherical with positive constant Tanaka-Webster curvature and vanishing pseudohermitian torsion. We also refer to [Ho] for another result related to this conjecture.

We remark that one of the difficulties is that CR Yamabe flow does not improve the behavior of the pseudohermitian torsion. So another flow, called the CR torsion flow, was considered by the second named author and his coauthors ([CKW]) on a pseudohermitian 3-manifold: the CR torsion flow is defined by

$$
\left\{\begin{array}{l}
\frac{\partial}{\partial t} J(t)=-2 J A_{J, \theta}(t) \\
\frac{\partial}{\partial t} \theta(t)=-2 W(t) \theta(t)
\end{array}\right.
$$

on $M \times[0, T)$ with the $\mathrm{CR}$ structure $J(t)=i \theta^{1} \otimes Z_{1}-i \theta^{\overline{1}} \otimes Z_{\overline{1}}$ and the pseudohermitian torsion tensor $A_{J, \theta}(t)=-i A_{11} \theta^{1} \otimes Z_{\overline{1}}+i A_{\overline{1} \overline{1}} \theta^{\overline{1}} \otimes Z_{1}$. This flow is a CR analogue of the Ricci flow, and the CR Yamabe flow (1.2) is a special formulation of it.

On the other hand, in view of the work of [DS], CSZ, and CMM], understanding the structure of CR Yamabe solitons may be a necessary step in approaching the asymptotic convergence of solutions of the CR Yamabe flow (1.2). Indeed, one expects CR Yamabe solitons to model singularity formations of the CR Yamabe flow.

In this paper, we investigate the geometry and classification of three-dimensional closed CR Yamabe solitons $\left(M^{3}, J, \theta, f, \mu\right)$ satisfying the following equivalent soliton 
equations

$$
\left\{\begin{array}{l}
W+\frac{1}{2} f_{0}=\mu \\
f_{11}+i A_{11} f=0
\end{array}\right.
$$

for some smooth function $f$ on a pseudohermitian 3-manifold $\left(M^{3}, J, \theta\right)$.

Our first result asserts that any closed three-dimensional CR Yamabe soliton must have constant Tanaka-Webster scalar curvature.

Theorem 1.1. If $\left(M^{3}, J, \theta, f, \mu\right)$ is a closed three-dimensional CR Yamabe soliton, then $\theta$ is the contact structure of constant Tanaka-Webster scalar curvature $\mu$, i.e., $f_{0}=0$.

Our second result establishes the vanishing of the pseudohermitian torsion of a closed three-dimensional CR Yamabe soliton under certain conditions.

Theorem 1.2. Let $\left(M^{3}, J, \theta, f, \mu\right)$ be a closed CR Yamabe soliton with $P_{0} f=0$ for the CR Paneitz operator $P_{0}$. If the contact class $[\theta]$ of $\theta$ admits a contact form $\tilde{\theta}$ of the vanishing $\mathrm{CR} \widetilde{Q}$-curvature, then $(M, J, \theta)$ is a closed pseudohermitian 3-manifold of vanishing pseudohermitian torsion provided that $f$ is nowhere vanishing.

Remark 1.2. (i). The kernel space of CR Paneitz operator $P_{0}$ is infinite-dimensional.

(ii). Since a pseudohermitian 3-manifold $(M, J, \theta)$ of constant Tanaka-Webster scalar curvature and vanishing pseudohermitian torsion must be spherical (see Definition 2.1 and Eq. (2.8) ), it follows from Theorem 1.1, Theorem 1.2 and a result of Y. Kamishima and T. Tsuboi ([KT]) that one can have a complete classification of such closed spherical torsion-free CR Yamabe solitons.

(iii). It is conjectured that any closed three-dimensional pseudohermitian manifold admits a contact form of vanishing CR $Q$-curvature (cf. [FH, CCC, CS]). Therefore, in view of Theorem 1.2 , we have

Conjecture 1.2. Any 3-dimensional closed CR Yamabe soliton $\left(M^{3}, J, \theta, f, \mu\right)$ with $P_{0} f=0$ and nowhere vanishing $f$ must have zero pseudohermitian torsion.

As a consequence of Theorem 1.2 and the work of J. Cao and the second named author $\mathrm{CaOC}$, we have the following classification of a special class of closed CR Yamabe solitons:

Corollary 1.1. Conjecture 1.2 holds if $\left(M^{3}, J, \theta\right)$ is the smooth boundary of a bounded strictly pseudoconvex domain $\Omega$ in a complete Stein manifold $V^{2}$.

In particular, if $M$ is a hypersurface in $\mathbb{C}^{2}$, i.e. $M=\partial \Omega$ for a bounded domain $\Omega$ in $\mathbb{C}^{2}$, then for any pluriharmonic function $u: \mathbf{U} \rightarrow \mathbb{R}(\partial \bar{\partial} u=0)$ with a simply connected $\mathbf{U} \subset \bar{\Omega}$, there exists a holomorphic function $w$ in $\mathbf{U}$ such that $u=\operatorname{Re}(w)$.

Now define $f:=\left.u\right|_{M}$, it follows that $f$ is a CR pluriharmonic function (see Definition 3.9) and

$$
P_{0} f=0
$$

Hence, we have

Corollary 1.2. Let $(M, J, \theta)$ be a smooth hypersurface in $\mathbb{C}^{2}$. If $\left(M^{3}, J, \theta, f, \mu\right)$ is a closed CR Yamabe soliton with $f:=\left.u\right|_{M}$ as above which vanishes nowhere. Then $(M, J, \theta)$ is a closed pseudohermitian 3-manifold of constant Tanaka-Webster scalar curvature $\mu$ and vanishing pseudohermitian torsion. 
Secondly, we consider complete three-dimensional pseudo-gradient CR Yamabe solitons defined as follows:

Definition 1.2. A complete pseudohermitian 3-manifold $\left(M^{3}, J, \theta\right)$ is called a complete pseudo-gradient CR Yamabe soliton if there exists a smooth function $\varphi$ on $M$ such that

$$
\left\{\begin{array}{l}
W+\frac{1}{2} \Delta_{b} \varphi=\mu \\
\varphi_{11}=0, \varphi_{0}=0
\end{array}\right.
$$

Again, it is called shrinking if $\mu>0$, steady if $\mu=0$, and expanding if $\mu<0$.

For example, by using Lemma 4.2, one can easily check that the Heisenberg group

$$
H^{3} \equiv\left\{(z, t) \in \mathbb{C} \times \mathbb{R} \mid\left(z_{1}, t_{1}\right) \cdot\left(z_{2}, t_{2}\right):=\left(z_{1}+z_{2}, t_{1}+t_{2}+\operatorname{Im}\left(z_{1} \overline{z_{2}}\right)\right)\right\},
$$

equipped with the standard CR structure and potential function $\varphi:=\mu|z|^{2}$, is a pseudogradient CR Yamabe soliton for any $\mu \in \mathbb{R}$. Since it is analogous to the Gaussian Ricci solitons defined on the Euclidean space $\mathbb{R}^{n}$ with the potential function $\mu|x|^{2}$, we call $\left(H^{3}, \mu|z|^{2}\right)$ a pseudo Gaussian soliton. Note that the Heisenberg group also admits CR Yamabe soliton structures of shrinking/steady/expanding type, as defined in (1.1), by taking $f=2 \mu t$.

For three-dimensional complete pseudo-gradient CR Yamabe solitons, motivated by the recent work [CSZ] of the first author with others on the structure of gradient Yamabe solitons, we explore the potential function $\varphi$ and investigate the geometry and topology of its level sets. It turns out that $\varphi$ is necessarily an isoparametric function which, according to [W], makes the critical set of $\varphi$ and regular level sets rather special (see Lemma 5.3 and Remark 5.1): the only singular level sets of $\varphi$ are the smooth focal varieties of $\varphi$ (possibly empty),

$$
\Sigma_{+}:=\left\{\varphi=\max _{x \in M} \varphi(x)\right\} \text { and } \Sigma_{-}:=\left\{\varphi=\min _{x \in M} \varphi(x)\right\},
$$

and each regular level set is a tube over either $\Sigma_{+}$or $\Sigma_{-}$. Furthermore, we prove that each regular level surface must have zero Gaussian curvature with respect to the induced metric of the Webster adapted metric on $M^{3}$. These special geometric and topological features allow us to conclude the possible diffeomorphism types of the underlying 3-manifold $M$.

Theorem 1.3. Let $\left(M^{3}, J, \theta, \varphi, \mu\right)$ be a non-trivial three-dimensional complete pseudogradient CR Yamabe soliton with vanishing torsion. Then the potential function $\varphi$ is an isoparametric function and $M$ is diffeomorphic to one of the following spaces:

$\mathbb{R}^{3}, \mathbb{S}^{3}, L(p, q), \mathbb{S}^{2} \times \mathbb{R}, \mathbb{S}^{1} \times \mathbb{R}^{2}, \mathbb{T}^{2} \times \mathbb{R}, \mathbb{T}^{2} \times[0, \infty)$ with $\mathbb{T}^{2} \times\{0\}$ collapsing to $\mathbb{S}^{1}$ More precisely, besides possible surface components, the critical set of $\varphi$ can contain at most two curves. Furthermore,

(i) if the critical set is empty or consists of only surfaces, then $M$ is diffeomorphic to either $\mathbb{R}^{3}$, or $\mathbb{T}^{2} \times \mathbb{R}$, or $S^{1} \times \mathbb{R}^{2}$, where $\mathbb{T}^{2}$ denotes the 2-torus;

(ii) if the critical set contains only one curve, then $M$ is diffeomorphic to either $\mathbb{R}^{3}$ or $\mathbb{T}^{2} \times[0, \infty)$ with $\mathbb{T}^{2} \times\{0\}$ collapsing to $S^{1}$;

(iii) if the critical set contains two curves, then $M$ is diffeomorphic to either $\mathbb{S}^{3}$, or $\mathbb{S}^{2} \times \mathbb{R}$, or the lens spaces $L(p, q)$ with $1 \leq q<p$.

Moreover, when $M^{3}$ is simply-connected, we can further show the following 
Corollary 1.3. Let $\left(M^{3}, J, \theta, \varphi, \mu\right)$ be a simply-connected complete pseudo-gradient CR Yamabe soliton with vanishing torsion and $W>\mu$ (or $W<\mu$ ), then it must be trivial or diffeomorphic to $\mathbb{R}^{3}$.

Remark 1.3. M. Rumin ([Ru $)$ has proven that a complete pseudohermitian 3-manifold of positive Tanaka-Webster scalar curvature and vanishing torsion must be compact. Hence, if $W \geq \mu>0$, then the soliton is trivial and $W=\mu$. In fact, the manifold is the CR 3-sphere (cf. [KT]).

The rest of the paper is organized as follows. In Section 2, we review some basic materials of pseudohermitian manifolds and recall some basic facts from the CR Yamabe flow. In Section 3, we first explain that CR Yamabe solitons correspond to self-similar solutions of the CR Yamabe flow and then prove Theorem 1.1, Theorem 1.2 and the two corollaries. Complete pseudo-gradient CR Yamabe solitons are defined in Section 4. Some basic properties are also derived there. In Section 5, we investigate the structure of pseudo-gradient CR Yamabe solitons with vanishing torsion.

Acknowledgements. We would like to thank Professor Feng Luo for very helpful discussions related to Theorem 1.3 and Professor Reiko Miyaoka for her comment during the 7th OCAMI-TIMS Workshop which led us to remove an extra assumption of Theorem 1.3 in an early version. The research of the first author was partially supported by the Science and Technology Development Fund (Macao S.A.R.) Grant FDCT/016/2013/A1, as well as RDG010 grant of University of Macau. Part of the project was done during the visit of the second and the third authors to the University of Macau in spring 2014. They would like to express their thanks to the institution for the warm hospitality.

\section{Preliminaries}

In this section, we first introduce some basic materials about pseudohermitian 3manifolds, and then collect some basic facts about the CR Yamabe flow. We refer the reader to $[\mathrm{CCW}]$ and $[\mathrm{CC}]$ for more details and [L1, L2] for higher dimensional cases.

Let $M$ be a complete 3-manifold with an oriented contact structure $\xi$. There always exists a global contact form $\theta$ with $\xi=\operatorname{ker} \theta$, obtained by patching together local ones with a partition of unity. The unique vector field $\mathbf{T}$ such that $\theta(\mathbf{T})=1$ and $L_{\mathbf{T}} \theta=0$ or $d \theta(\mathbf{T}, \cdot)=0$ is called the Reeb vector field of $\theta$. A CR structure compatible with $\xi$ is a smooth endomorphism $J: \xi \rightarrow \xi$ such that $J^{2}=-I d$. A pseudohermitian structure compatible with $\xi$ is a CR-structure $J$ compatible with $\xi$ together with a global contact form $\theta$. The CR structure $J$ extends to $\mathbb{C} \otimes \xi$ and decomposes $\mathbb{C} \otimes \xi$ into the direct sum of $T_{1,0}$ and $T_{0,1}$ which are eigenspaces of $J$ with respect to eigenvalues $i$ and $-i$, respectively.

Let $\left\{\mathbf{T}, Z_{1}, Z_{\overline{1}}\right\}$ be a frame of $T M \otimes \mathbb{C}$, where $Z_{1} \in T_{1,0}$ and $Z_{\overline{1}}=\overline{Z_{1}} \in T_{0,1}$, and $\left\{\theta, \theta^{1}, \theta^{\overline{1}}\right\}$ be the coframe dual to it. Since $d \theta(\mathbf{T}, \cdot)=0$, there exists a positive function $h_{1 \overline{1}}$ such that

$$
d \theta=i h_{1 \overline{1}} \theta^{1} \wedge \theta^{\overline{1}} .
$$

In this article, we always normalize $Z_{1}$ such that $h_{1 \overline{1}}=1$ and denote $d \mu:=\theta \wedge d \theta$ as the volume form of $M$. 
Now we introduce the Levi form $\langle,\rangle_{L_{\theta}}$, which is defined by

$$
\langle V, W\rangle_{L_{\theta}}=-i d \theta(V \wedge \bar{W})=d \theta(V \wedge J \bar{W}),
$$

where $V, W \in T_{1,0}$. By defining $\langle\bar{V}, \bar{W}\rangle_{L_{\theta}}=\overline{\langle V, W\rangle_{L_{\theta}}}$ for all $V, W \in T_{1,0}$, one can extend the Levi form to $T_{0,1}$. In fact, the Levi form can be further extended as a hermitian form on all the tensor bundles composed of $T_{0,1}, T_{1,0}$ and their duals.

Let $\theta_{1}{ }^{1}$ be the unique purely imaginary 1 -form such that

$$
d \theta^{1}=\theta^{1} \wedge \theta_{1}^{1}+\theta \wedge \tau^{1}
$$

where $\tau^{1}=A^{1} \overline{1}^{\overline{1}}$ is the pseudohermitian torsion. Then the 1 -forms $\theta_{1}{ }^{1}$ and $\theta_{\overline{1}}{ }^{\overline{1}}:=\overline{\theta_{1}{ }^{1}}$, as connection coefficients, characterize the pseudohermitian connection $\nabla$ of $(M, J, \theta)$. Namely,

$$
\nabla Z_{1}=\theta_{1}^{1} \otimes Z_{1}, \quad \nabla Z_{\overline{1}}=\theta_{\overline{1}}{ }^{\overline{1}} \otimes Z_{\overline{1}} \quad \text { and } \quad \nabla \mathbf{T}=0 .
$$

Note that $\nabla$ can be extended to all tensors naturally. Furthermore, we have the following structure equation

$$
d \theta_{1}{ }^{1}=W \theta^{1} \wedge \theta^{\overline{1}}+2 i \operatorname{Im}\left(A_{1, \overline{1}}^{\overline{1}} \theta^{1} \wedge \theta\right)
$$

and $W$ is called the Tanaka-Webster curvature. Here we denote components of covariant derivatives with indices preceded by comma; thus write $A_{1, \overline{1}}^{\overline{1}} \theta^{1} \wedge \theta$. The indices $\{0,1, \overline{1}\}$ indicate derivatives with respect to $\left\{\mathbf{T}, Z_{1}, Z_{\overline{1}}\right\}$. For derivatives of a scalar function, we often omit the comma, for instance, $\varphi_{1}=Z_{1} \varphi, \varphi_{1 \overline{1}}=Z_{\overline{1}} Z_{1} \varphi-$ $\theta_{1}{ }^{1}\left(Z_{\overline{1}}\right) Z_{1} \varphi, \varphi_{0}=\mathbf{T} \varphi$ for a (smooth) function $\varphi$.

For a real-valued function $\varphi$, the subgradient $\nabla_{b}$ is defined by $\nabla_{b} \varphi \in \xi$ and $\left\langle V, \nabla_{b} \varphi\right\rangle_{L_{\theta}}=$ $d \varphi(V)$ for all vector fields $V$ tangent to contact plane. Locally $\nabla_{b} \varphi=\varphi_{\overline{1}} Z_{1}+\varphi_{1} Z_{\overline{1}}$. We can use the connection to define the subhessian as the complex linear map $\left(\nabla^{H}\right)^{2} \varphi$ : $T_{1,0} \oplus T_{0,1} \rightarrow T_{1,0} \oplus T_{0,1}$ with

$$
\left(\nabla^{H}\right)^{2} \varphi(V)=\nabla_{V} \nabla_{b} \varphi
$$

The sub-Laplacian $\Delta_{b}$ is defined to be the trace of the subhessian

$$
\Delta_{b} \varphi=\operatorname{tr}\left(\left(\nabla^{H}\right)^{2} \varphi\right)=\varphi_{1 \overline{1}}+\varphi_{\overline{1} 1} .
$$

For all $V=V^{1} Z_{1} \in T_{1,0}$, we define

$$
\begin{aligned}
& \operatorname{Ric}(V, V)=W V^{1} V^{\overline{1}}=W|V|_{L_{\theta}}^{2}, \\
& \operatorname{Tor}(V, V)=2 \operatorname{Re} i A_{\overline{1} \overline{1}} V^{\overline{1}} V^{\overline{1}} .
\end{aligned}
$$

We recall the following commutation relations ([L1]).

$$
\begin{aligned}
& C_{I, 01}-C_{I, 10}=C_{I, \overline{1}} A_{11}-k C_{I,} A_{11, \overline{1}}, \\
& C_{I, 0 \overline{1}}-C_{I, \overline{1} 0}=C_{I, 1} A_{\overline{1} \overline{1}}+k C_{I,} A_{\overline{1} \overline{1}, 1}, \\
& C_{I, 1 \overline{1}}-C_{I, \overline{1} 1}=i C_{I, 0}+k W C_{I},
\end{aligned}
$$

here $C_{I}$ denotes a coefficient of a tensor with multi-index $I$ consisting of only 1 and $\overline{1}$, and $k$ is the number of 1 's minus the number of $\overline{1}$ 's in $I$.

Next we rewrite the purely imaginary 1 -forms $\theta_{1}{ }^{1}=i \sigma_{1}^{2}$ and $\theta_{\overline{1}}{ }^{\overline{1}}=i \sigma_{2}^{1}$ in terms of real 1 -forms $\sigma_{1}^{2}$ and $\sigma_{2}^{1}$ with $\sigma_{2}^{1}=-\sigma_{1}^{2}$ by (2.2). Let $Z_{1}=\frac{1}{2}\left(e_{1}-i e_{2}\right)$ for real vectors $e_{1}$ and $e_{2}$. It follows that $e_{2}=J e_{1}$. Let $e^{1}=\operatorname{Re}\left(\theta^{1}\right)$ and $e^{2}=\operatorname{Im}\left(\theta^{1}\right)$. Then $\left\{e^{1}, e^{2}, \theta\right\}$ is 
dual to $\left\{e_{1}, e_{2}, \mathbf{T}\right\}$. Now in view of (2.2) and (2.1), we have the following real version of structure equations ([CChi $]$ ) :

$$
\begin{aligned}
d \theta & =2 e^{1} \wedge e^{2}, \\
\nabla_{b} e_{1} & =\sigma_{1}^{2} \otimes e_{2}, \quad \nabla_{b} e_{2}=\sigma_{2}^{1} \otimes e_{1}, \\
d e^{1} & =e^{2} \wedge \sigma_{2}^{1} \bmod \theta ; d e^{2}=e^{1} \wedge \sigma_{1}^{2} \bmod \theta .
\end{aligned}
$$

We also write $\varphi_{e_{i}}=e_{i} \varphi$ and $\nabla_{b} \varphi=\frac{1}{2}\left(\varphi_{e_{1}} e_{1}+\varphi_{e_{2}} e_{2}\right)$. Moreover we have

$$
\varphi_{e_{i} e_{j}}=e_{j} e_{i} \varphi-\sigma_{i}^{k}\left(e_{j}\right) \varphi_{e_{k}}
$$

for $i, j, k=1,2$ and

$$
\Delta_{b} \varphi=\left(\varphi_{1 \overline{1}}+\varphi_{\overline{1} 1}\right)=\frac{1}{2}\left(\varphi_{e_{1} e_{1}}+\varphi_{e_{2} e_{2}}\right) .
$$

The real version of the commutation relations are

$$
\begin{array}{ll}
\varphi_{e_{1} e_{2}}-\varphi_{e_{2} e_{1}} & =2 \varphi_{0} \\
\varphi_{0 e_{1}}-\varphi_{e_{1} 0} & =\varphi_{e_{1}} \operatorname{Re} A_{11}-\varphi_{e_{2}} \operatorname{Im} A_{11} \\
\varphi_{0 e_{2}}-\varphi_{e_{2} 0} & =\varphi_{e_{1}} \operatorname{Im} A_{11}+\varphi_{e_{2}} \operatorname{Re} A_{11} \\
\varphi_{e_{1} e_{1} e_{2}}-\varphi_{e_{1} e_{2} e_{1}} & =2 \varphi_{e_{1} 0}-2 \varphi_{e_{2}} W \\
\varphi_{e_{2} e_{1} e_{2}}-\varphi_{e_{2} e_{2} e_{1}} & =2 \varphi_{e_{2} 0}+2 \varphi_{e_{1}} W .
\end{array}
$$

We also recall some definitions for later purposes.

Definition 2.1 ([CL, $[\mathrm{KT}])$. Let $(M, J, \theta)$ be a three-dimensional pseudohermitian manifold. Then the followings are equivalent:

(i) Its Cartan curvature tensor vanishes, i.e.,

$$
Q_{11}=\frac{1}{6} W_{11}+\frac{i}{2} W A_{11}-A_{11,0}-\frac{2 i}{3} A_{11, \overline{1} 1}=0 .
$$

(ii) It is locally $\mathrm{CR}$ equivalent to the standard pseudohermitian 3 -sphere $\left(\mathbf{S}^{3}, \widehat{J}, \widehat{\theta}\right)$.

If such properties hold, then we call the CR structure $J$ spherical and $(M, J, \theta)$ a spherical pseudohermitian 3-manifold. In particular, the spherical structure is CR invariant.

Definition 2.2. Let $(M, J, \theta)$ be a three-dimensional pseudohermitian manifold without boundary. A piecewise smooth curve $\gamma:[0,1] \rightarrow M$ is said to be a Legendrian curve if $\gamma^{\prime}(t) \in \xi$ whenever $\gamma^{\prime}(t)$ exists. The length of $\gamma$ is then defined by

$$
l(\gamma)=\int_{0}^{1} h\left(\gamma^{\prime}(t), \gamma^{\prime}(t)\right)^{\frac{1}{2}} d t
$$

where $h(X, Y)=d \theta(X, J Y)$. The Carnot-Carathéodory distance $d_{c}$ between any two points $p, q \in M$ is defined by

$$
d_{c}(p, q)=\inf \left\{l(\gamma) \mid \gamma \in C_{p, q}\right\},
$$

where $C_{p, q}$ is the set of all Legendrian curves which join $p$ and $q$. By Chow's connectivity theorem [Cho], for any two points $p, q \in M$, there always exists a Legendrian curve joining $p$ and $q$, so the distance is finite. We say $(M, J, \theta)$ is complete if $\left(M, d_{c}\right)$ is a complete metric space. 
Finally, in the following, we recall some basic facts about the CR Yamabe flow (1.2) on a closed pseudohermitian 3 -manifold $\left(M^{3}, J, \stackrel{\circ}{\theta}\right)$ with $\theta(0)=\stackrel{\circ}{\theta}$. From $([\mathrm{CC}])$, we have

Lemma 2.1. Let $\left(M^{3}, J, \stackrel{\circ}{\theta}\right)$ be a closed pseudohermitian 3-manifold. Under the CR Yamabe flow (1.2), we have

$$
\frac{\partial}{\partial t} W=4 \Delta_{b} W+2 W^{2}
$$

and

$$
\frac{\partial}{\partial t} A_{11}=2 W A_{11}-2 i W_{11}
$$

Here $\Delta_{b}, A_{11}$ and the covariant derivatives are with respect to $\theta(x, t)$ which is in the contact class $[\stackrel{\circ}{\theta}(x)]$ with $\theta(x, 0)=\stackrel{\theta}{\theta}(x)$.

Now it follows from applying the maximum principle to (2.9) that

Lemma 2.2. Let $\left(M^{3}, J, \stackrel{\circ}{\theta}\right)$ be a closed pseudohermitian 3-manifold with positive

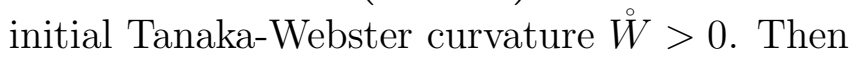

$$
W(t)>0
$$

is preserved under the CR Yamabe flow (1.2).

Moreover, if $J$ is spherical so that

$$
Q_{11}=\frac{1}{6} W_{11}+\frac{i}{2} W A_{11}-A_{11,0}-\frac{2 i}{3} A_{11, \overline{1} 1}=0,
$$

i.e.,

$$
\frac{1}{6} W_{11}=-\frac{i}{2} W A_{11}+A_{11,0}+\frac{2 i}{3} A_{11, \overline{1} 1},
$$

then, by using this and the commutation relation, one can rewrite the RHS of (2.10) as

$$
4\left(A_{11, \overline{1} 1}+A_{11,1 \overline{1}}-4 i A_{11,0}\right)-12 W A_{11} .
$$

Thus, we have

$$
\frac{\partial}{\partial t} A_{11}=-4 \mathcal{L}_{4} A_{11}-12 W A_{11}
$$

so the highest "weight" term is just -4 times the generalized Folland-Stein operator

$$
\mathcal{L}_{\alpha}=-\Delta_{b}+i \alpha \mathbf{T}
$$

acting on $A_{11}$ with $\alpha=4$. Since $\alpha=4$ is clearly not an odd integer, $-\mathcal{L}_{4}$ is subelliptic on a closed pseudohermitian 3-manifold. It follows from the standard theory for subelliptic equations that we have

Lemma 2.3. Let $\left(M^{3}, J, \stackrel{\circ}{\theta}\right)$ be a closed spherical pseudohermitian 3-manifold with vanishing initial torsion $\AA_{11}=0$. Then

$$
A_{11}(t)=0
$$

is preserved under the CR Yamabe flow (1.2). 


\section{The Structure of Closed three-dimensional CR Yamabe Solitons}

In this section, by using the Harnack quantity derived in (3.7), we show that every closed three-dimensional CR Yamabe soliton has constant Tanaka-Webster curvature.

We first recall a result from [G] (see also [CL], Lemma 3.4 and Lemma 3.5).

Lemma 3.1. Let $\left(M^{2 n+1}, J, \theta\right)$ be a pseudohermitian $2 n+1$-manifold. For any smooth function $f$ on $M$, let $X_{f}$ be the vector field uniquely defined by

$$
\left.X_{f}\right\rfloor d \theta=d f \bmod \theta
$$

and

$$
\left.X_{f}\right\rfloor \theta=-f .
$$

Then

$$
X_{f}=i f_{\alpha} Z_{\bar{\alpha}}-i f_{\bar{\alpha}} Z_{\alpha}-f \mathbf{T}
$$

and it is a smooth infinitesimal contact diffeomorphism of $\left(M^{2 n+1}, \theta\right)$. Conversely, every smooth infinitesimal contact diffeomorphism is of the form $X_{f}$ for some smooth function $f$. Moreover, $L_{X_{f}} J$ has the following expression

$$
L_{X_{f}} J \equiv 2\left(f_{\alpha \alpha}+i A_{\alpha \alpha} f\right) \theta^{\alpha} \otimes Z_{\bar{\alpha}}+2\left(f_{\bar{\alpha} \bar{\alpha}}-i A_{\bar{\alpha} \bar{\alpha}} f\right) \theta^{\bar{\alpha}} \otimes Z_{\alpha} \bmod \theta .
$$

Remark 3.1. Note that, since $L_{\mathbf{T}} \theta=0, L_{X_{f}-c \mathbf{T}} \theta=\lambda \theta$ for any constant $c$ if $L_{X_{f}} \theta=\lambda \theta$. Hence if $X_{f}$ is an infinitesimal contact diffeomorphism, so is $X_{\tilde{f}}:=X_{f}-c \mathbf{T}$ with $\tilde{f}=f+c$.

Definition 3.1. A pseudohermitian 3-manifold $\left(M^{3}, J, \theta\right)$ is called a CR Yamabe soliton if there exist a function $f$ and a constant $\mu$ such that

$$
W+\frac{1}{2} f_{0}=\mu
$$

and

$$
f_{11}+i A_{11} f=0 .
$$

It is called shrinking if $\mu>0$, steady if $\mu=0$, and expanding if $\mu<0$. The equation (3.5) is corresponding to the CR vector field condition as in (3.6) below.

Next, we relate CR Yamabe solitons and self-similar solutions to the CR Ymamabe flow. A special class of solutions to the CR Yamabe flow (1.2) is given by self-similar solutions, whose contact forms $\theta_{t}$ deform under the CR Yamabe flow only by a scaling function depending on $t$ and reparametrizations by a 1-parameter family of contact diffeomorphisms; meanwhile, the CR structure $J$ shall be invariant under these diffeomorphisms.

Precisely, let $\Phi_{t}: M \rightarrow M$ be a one-parameter family of contact diffeomorphisms generated by a CR vector field $X_{\tilde{f}}$ as above on $M$ with $\Phi_{0}=i d_{M}$. Set

$$
\theta_{t}:=\rho(t) \Phi_{t}^{*} \stackrel{\circ}{\theta}, \quad \rho(t)>0, \quad \rho(0)=1 .
$$

Then $\left(M, J, \theta_{t}\right)$ is a self-similar solution to the CR Yamabe flow (1.2) whenever

$$
-2 W \theta_{t}=\frac{\partial}{\partial t} \theta_{t}=\rho^{\prime}(t) \Phi_{t}^{*} \stackrel{\circ}{\theta}+\rho(t) \Phi_{t}^{*}\left(L_{X_{\tilde{f}}} \stackrel{\circ}{)}\right)=(\log \rho)^{\prime}(t) \theta_{t}+L_{X_{\tilde{f}}} \theta_{t} .
$$


It follows from (3.1) and $d \theta(\mathbf{T}, \cdot)=0$ that

$$
\left.X_{\tilde{f}}\right\rfloor d \theta_{t}=d \tilde{f}-\tilde{f}_{0} \theta_{t}
$$

and then

$$
\left.\left.\left.L_{X_{\tilde{f}}} \theta_{t}=X_{\widetilde{f}}\right\rfloor d \theta_{t}+d\left(X_{\widetilde{f}}\right\rfloor \theta_{t}\right)=X_{f}\right\rfloor d \theta_{t}-d \widetilde{f}=-\widetilde{f}_{0} \theta_{t}
$$

Hence,

$$
-2 W \theta_{t}-(\log \rho)^{\prime}(t) \theta_{t}=L_{X_{\tilde{f}}} \theta_{t}=-\widetilde{f}_{0} \theta_{t} .
$$

In short, every self-similar solution satisfies the equation

$$
2 W \theta_{t}+L_{X_{\tilde{f}}} \theta_{t}=2 W \theta_{t}-\widetilde{f}_{0} \theta_{t}=-(\log \rho)^{\prime}(t) \theta_{t}
$$

for some scaling function $\rho(t)>0$ and potential function $\tilde{f}: M \rightarrow \mathbb{R}$. When $t=0$, we obtain the equation

$$
W-\frac{1}{2} \widetilde{f}_{0}=-\frac{1}{2} \rho^{\prime}(0)
$$

for $(M, J, \theta)$. If we put $f=-\tilde{f}$ and denote $\mu=-\frac{1}{2} \rho^{\prime}(0)$ then we have

$$
W+\frac{1}{2} f_{0}=\mu \text {. }
$$

Recall that we still need to make sure $J$ is invariant under the contact diffeomorphism, i.e.,

$$
0=L_{X_{f}} J \equiv 2\left(f_{11}+i A_{11} f\right) \theta^{1} \otimes Z_{\overline{1}}+2\left(f_{\overline{1} \overline{1}}-i A_{\overline{1} \overline{1}} f\right) \theta^{\overline{1}} \otimes Z_{1} \bmod \theta .
$$

Thus $f_{11}+i A_{11} f=0$. (We refer to [CL], p. 240, for a proof of the expression of $L_{X_{f}} J$.)

Conversely, starting from a CR Yamabe soliton $(M, J, \stackrel{\circ}{\theta}, f, \mu)$ which satisfies $W+$ $\frac{1}{2} f_{0}=\mu$ and $f_{11}+i A_{11} f=0$, one can solve equations

$$
\left\{\begin{aligned}
\left.\frac{\partial}{\partial t} \rho(t)\right|_{t=0} & =-2 \mu, \\
\rho(0) & =1
\end{aligned}\right.
$$

and for $f(t):=f \circ \Phi_{t}$

$$
\left\{\begin{aligned}
\frac{\partial}{\partial t} \Phi_{t} & =X_{f(t)} \\
\Phi_{0} & =i d_{M}
\end{aligned}\right.
$$

to get $\rho(t)$ and $\Phi_{t}$, and thus a self-similar solution $\theta_{t}=\rho(t) \Phi_{t}^{*} \stackrel{\circ}{\text { of }}$ the CR Yamabe flow. Note that it suffices to take $\rho(t)=1-2 \mu t$. This would be the unique solution once we establish the uniqueness for CR Yamabe flow. The same phenomenon occurred in the theory of Ricci solitons (cf. CLN]).

In this article, we focus on CR Yamabe solitons instead of their corresponding selfsimilar solutions.

To prove Theorem 1.1, we shall need a certain differential Harnack quantity for the three-dimensional CR Yamabe flow. We remark that in the Ricci flow, Hamilton found a conserved quantity which vanishes identically for expanding Ricci solitons and showed that such a quantity is nonnegative for generic solutions with positive curvature operator. This quantity is called the differential Harnack quantity, or Li-Yau-Hamilton (LYH) quantity. In the paper [CC], J.-H. Cheng and the second named author have indicated a similar Harnack quantity for the CR Yamabe flow (1.2). For the reader's convenience, we give a detailed proof here. 
Lemma 3.2. A three-dimensional CR Yamabe soliton satisfies:

$$
4 \Delta_{b} W+2 W(W-\mu)-W_{0} f-\left\langle\nabla_{b} W, J\left(\nabla_{b} f\right)\right\rangle_{\theta}=0,
$$

Proof. Recall that $\Delta_{b} W=W_{1 \overline{1}}+W_{\overline{1} 1}$. We first differentiate the soliton equation (3.4) and obtain

$$
W_{1 \overline{1}}=-\frac{1}{2} f_{01 \overline{1}}=\frac{i}{2}\left(f_{1 \overline{1}}-f_{\overline{1} 1}\right)_{1 \overline{1}} .
$$

The two terms appear on the right hand side are higher derivatives of $f$ which can be reduced by using the $\mathrm{CR}$ vector field condition

$$
f_{11}+i A_{11} f=0 .
$$

Indeed, by using commutation relations (2.4), one derives

$$
\begin{aligned}
f_{1 \overline{1} 1} & =f_{11 \overline{1}}-i f_{10}-f_{1} W \\
& =-i\left(A_{11} f\right)_{\overline{1}}-i f_{01}+i A_{11} f_{\overline{1}}-f_{1} W \\
& =-i A_{11, \overline{1}} f+2 i W_{1}-f_{1} W .
\end{aligned}
$$

Differentiate it in the direction $Z_{\overline{1}}$, one achieves

$$
f_{1 \overline{1} 1 \overline{1}}=-i A_{11, \overline{1} \overline{1}} f-i A_{11, \overline{1}} f_{\overline{1}}+2 i W_{1 \overline{1}}-f_{1 \overline{1}} W-f_{1} W_{\overline{1}} .
$$

On the other hand, the differentiation of its conjugation in the $Z_{1}$ direction gives an expression of $f_{\overline{1} 1 \overline{1} 1}$. After changing the $3 \mathrm{rd}$ and 4 th indices, one obtains

$$
\begin{aligned}
f_{\overline{1} 11 \overline{1}} & =f_{\overline{1} 1 \overline{1} 1}+i f_{\overline{1} 10} \\
& =i A_{\overline{1} \overline{1}, 11} f+i A_{\overline{1} \overline{1}, 1} f_{1}-2 i W_{\overline{1} 1}-f_{\overline{1} 1} W-f_{\overline{1}} W_{1}+i f_{\overline{1} 10},
\end{aligned}
$$

where we can further reduce the bad term $f_{\overline{1} 10}$ by noticing that

$$
\begin{aligned}
f_{\overline{1} 10} & =f_{0 \overline{1} 1}-A_{11} f_{\overline{1} \overline{1}}-A_{11, \overline{1}} f_{\overline{1}}-A_{\overline{1} \overline{1}} f_{11}-A_{\overline{1} \overline{1}, 1} f_{1} \\
& =-2 W_{\overline{1} 1}-A_{11} f_{\overline{1} \overline{1}}-A_{11, \overline{1}} f_{\overline{1}}-A_{\overline{1} \overline{1}} f_{11}-A_{\overline{1} \overline{1}, 1} f_{1} \\
& =-2 W_{\overline{1} 1}-A_{11, \overline{1}} f_{\overline{1}}-A_{\overline{1} \overline{1}, 1} f_{1} .
\end{aligned}
$$

Substituting our expressions for $f_{1 \overline{1} 1 \overline{1}}$ and $f_{\overline{1} 11 \overline{1}}$ into the equation for $W_{1 \overline{1}}$, we get

$$
\begin{aligned}
2 W_{1 \overline{1}}= & A_{11, \overline{1} \overline{1}} f+A_{11, \overline{1}} f_{\overline{1}}-2 W_{1 \overline{1}}-i f_{1 \overline{1}} W-i f_{1} W_{\overline{1}} \\
& +A_{\overline{1} \overline{1}, 11} f+A_{\overline{1} \overline{1}, 1} f_{1}-2 W_{\overline{1} 1}+i f_{\overline{1} 1} W+i f_{\overline{1}} W_{1} \\
& -2 W_{\overline{1} 1}-A_{11, \overline{1}} f_{\overline{1}}-A_{\overline{1} \overline{1}, 1} f_{1} .
\end{aligned}
$$

Finally, by the CR Bianchi identity $A_{11, \overline{1} \overline{1}}+A_{\overline{1} \overline{1}, 11}=W_{0}$, we have the Harnack quantity

$$
\begin{aligned}
4 \Delta_{b} W & =\left(A_{11, \overline{1} \overline{1}}+A_{\overline{1} \overline{1}, 11}\right) f-i\left(f_{1 \overline{1}}-f_{\overline{1} 1}\right) W-i\left(f_{1} W_{\overline{1}}-f_{\overline{1}} W_{1}\right) \\
& =W_{0} f-i\left(i f_{0}\right) W-i\left(f_{1} W_{\overline{1}}-f_{\overline{1}} W_{1}\right) \\
& =-2 W(W-\mu)+W_{0} f+\left\langle\nabla_{b} W, J\left(\nabla_{b} f\right)\right\rangle_{\theta} .
\end{aligned}
$$

This finishes the proof of (3.7).

Now by using the Harnack quantity (3.7), we can show that every closed CR Yamabe soliton has constant Tanaka-Webster curvature. This computation is similar to the one given by S.-Y. Hsu [Hsu] for closed Riemannian Yamabe solitons.

The Proof of Theorem 1.1: 
Proof. Integrating the Harnack quantity (3.7), one derives that

$$
\begin{aligned}
0 & =\int_{M}\left[-2 W(W-\mu)+W_{0} f-i\left(f_{1} W_{\overline{1}}-f_{\overline{1}} W_{1}\right)\right] d \mu \\
& =-2 \int_{M} W(W-\mu) d \mu-\int_{M} W f_{0} d \mu+\int_{M} i\left(f_{1 \overline{1}}-f_{\overline{1} 1}\right) W d \mu \\
& =-2 \int_{M} W(W-\mu) d \mu-2 \int_{M} W f_{0} d \mu \\
& =-2 \int_{M} W(W-\mu) d \mu+4 \int_{M} W(W-\mu) d \mu \\
& =2 \int_{M} W(W-\mu) d \mu .
\end{aligned}
$$

Together with the fact that $\int_{M}(W-\mu) d \mu=-\frac{1}{2} \int_{M} f_{0} d \mu=\frac{1}{2} \int_{M} f \cdot \operatorname{div} \mathbf{T} d \mu=0$, we obtain

$$
\int_{M}(W-\mu)^{2} d \mu=\int_{M} W(W-\mu) d \mu-\mu \int_{M}(W-\mu) d \mu=0
$$

Next, to prepare the proof of Theorem 1.2, we first recall the definitions of CR Paneitz operator and the CR $Q$-curvature:

Definition 3.2. Let $(M, J, \theta)$ be a closed 3-dimensional pseudohermitian manifold. We define, as in [L1], that

$$
P \varphi:=\left(P_{1} \varphi\right) \theta^{1}:=\left(\varphi_{1}^{{ }_{1}}{ }_{1}+i A_{11} \varphi^{1}\right) \theta^{1}
$$

and

$$
\bar{P} \varphi:=\left(\bar{P}_{1}\right) \theta^{\overline{1}} .
$$

The operator $P$ characterizes CR-pluriharmonic functions. The CR Paneitz operator $P_{0}$ is defined by

$$
P_{0} \varphi=\left(\delta_{b}(P \varphi)+\bar{\delta}_{b}(\bar{P} \varphi)\right),
$$

where $\delta_{b}$ is the divergence operator that takes $(1,0)$-forms to functions by $\delta_{b}\left(\sigma_{1} \theta^{1}\right)=$ $\sigma_{1}{ }^{1}$, and similarly, $\bar{\delta}_{b}\left(\sigma_{\overline{1}} \theta^{\overline{1}}\right)=\sigma_{\overline{1}}{ }^{\overline{1}}$. One can check that $P_{0}$ is self-adjoint, that is, $\left\langle P_{0} \varphi, \psi\right\rangle=\left\langle\varphi, P_{0} \psi\right\rangle$ for all smooth functions $\varphi$ and $\psi$. For the details about these operators, the reader can consult [GL], [Hi], [L1], and [CChi].

Definition 3.3. ([Hi, $\mathrm{CCC}]$ ) Define the tensor

$$
R_{1} \theta^{1}:=\left(W_{1}-i A_{11, \overline{1}}\right) \theta^{1} .
$$

Use this to define the CR $Q$-curvature on $\left(M^{3}, \theta\right)$ by

$$
Q:=-c R_{1, \overline{1}}=-\frac{c}{2}\left(\Delta_{b} W+2 \operatorname{Im} A_{11, \overline{1} \overline{1}}\right),
$$

for some constant $c$. The last equality follows from [Hi, Lemma 5.4]. Clearly, vanishing of $R_{1}$ guarantees vanishing of the $\mathrm{CR} Q$-curvature.

Lemma 3.3. ([Hi, Lemma 5.4]) Let $\left(M^{3}, J, \theta\right)$ be a pseudohermitian manifold. Then, for rescaled contact form $\widetilde{\theta}=e^{2 g} \theta$, we have

$$
\widetilde{R}_{1}=e^{-3 g}\left(R_{1}-6 P_{1} g\right)
$$

and thus

$$
\widetilde{R}_{1, \overline{1}}=e^{-4 g}\left(R_{1, \overline{1}}-6 C_{\theta} g\right), \quad C_{\theta} g=\left(P_{1} g\right)_{\overline{1}} .
$$

Corollary 3.1. Let $\left(M^{3}, J, \theta\right)$ be a pseudohermitian manifold. If $\widetilde{\theta}=e^{2 g} \theta$ has vanishing CR $\widetilde{Q}$-curvature for some function $g$, then

$$
\Delta_{b} W+2 \operatorname{Im} A_{11, \overline{1} \overline{1}}=12 C_{\theta} g .
$$


Proof. The result follows from (3.13) and (3.11) easily as

$$
0=R_{1, \overline{1}}-6 C_{\theta} g=\frac{1}{2}\left(\Delta_{b} W+2 \operatorname{Im} A_{11, \overline{1} \overline{1}}\right)-6 C_{\theta} g .
$$

\section{The Proof of Theorem 1.2 :}

Proof. It follows from the CR vector field condition (3.5) that

$$
0=\int_{M}\left(A_{\overline{1} \overline{1}} f_{11}+i\left|A_{11}\right|^{2} f\right) d \mu=\int_{M}\left(A_{\overline{1} \overline{1}, 11} f\right) d \mu+i \int_{M}\left|A_{11}\right|^{2} f d \mu
$$

and

$$
0=\int_{M}\left(A_{11} f_{\overline{1} \overline{1}}-i\left|A_{11}\right|^{2} f\right) d \mu=\int_{M}\left(A_{11, \overline{1} \overline{1}} f\right) d \mu-i \int_{M}\left|A_{11}\right|^{2} f d \mu
$$

Thus,

$$
0=\int_{M}\left(\operatorname{Im} A_{11, \overline{1} \overline{1}} f\right) d \mu-\int_{M}\left|A_{11}\right|^{2} f d \mu .
$$

Now since the closed CR Yamabe soliton has constant Tanaka-Webster scalar curvature, from Corollary 3.1 we have

$$
\operatorname{Im} A_{11, \overline{1} \overline{1}}=6 C_{\theta} g .
$$

This together with (3.14) implies that

$$
\int_{M}\left|A_{11}\right|^{2} f d \mu=\int_{M}\left(\operatorname{Im} A_{11, \overline{1} \overline{1}} f\right) d \mu=6 \int_{M}\left(C_{\theta} g\right) f d \mu=3 \int_{M}\left(P_{0} g\right) f d \mu=3 \int_{M}\left(P_{0} f\right) g d \mu=0 .
$$

Here $P_{0} g=C_{\theta} g+\overline{C_{\theta} g}=2 C_{\theta} g$. Since $f$ vanishes nowhere, we obtain

$$
A_{11}=0 .
$$

\section{The Proof of Corollary 1.1:}

Proof. In the paper [CaoC], J. Cao and the second named author proved that if $(M, J, \theta)$ is the smooth boundary of a bounded strictly pseudoconvex domain $\Omega$ in a complete Stein manifold $V^{2}$, then there exists a smooth function $g$ such that $\widetilde{\theta}=e^{2 g} \theta$ has vanishing CR $\widetilde{Q}$-curvature. Hence, Corollary 1.1 follows from Theorem 1.2 .

\section{Complete 3-dimensional Pseudo-Gradient CR Yamabe Solitons}

We first recall that the family of Webster adapted metrics $h^{\lambda}$ of $(M, J, \theta)$ is given as the following:

$$
h^{\lambda}=\frac{1}{2} h+\lambda^{-2} \theta^{2}, \lambda>0
$$

with

$$
h(X, Y)=d \theta(X, J Y) .
$$

In this section, we discuss basic properties of complete CR Yamabe pseudo-gradient solitons, which are analogue to gradient Ricci solitons. Using these properties, we obtain the structure theorem for nontrivial complete 3-dimensional pseudo-gradient CR Yamabe solitons in the next section. 
Definition 4.1. A complete pseudohermitian 3-manifold $\left(M^{3}, J, \theta\right)$ is called a pseudogradient CR Yamabe soliton if there exist a smooth function $\varphi$ and $\mu \in \mathbb{R}$ such that

$$
W+\frac{1}{2} \Delta_{b} \varphi=\mu
$$

with

$$
\varphi_{11}=0 \quad \text { and } \quad \varphi_{0}=0 .
$$

$\left(M^{3}, J, \theta\right)$ is said to be trivial if $\varphi$ is a constant function.

Remark 4.1. (i) By Theorem 1.1, it is easy to see that a closed CR Yamabe soliton is always a trivial pseudo-gradient soliton. However, given a non-compact CR Yamabe soliton $(M, J, \theta, f, \mu)$, there might not even exist a function $\varphi$ such that $\Delta_{b} \varphi=f_{0}$.

(ii) The constraint $\varphi_{0}=0$ arises naturally when one requires the following equivalent expression of (4.1) to hold (cf. the proof of Lemma 4.1 (i)):

$$
R_{1 \overline{1}}+\varphi_{1 \overline{1}}=\mu h_{1 \overline{1}} .
$$

Here the positive function $h_{1 \overline{1}}$ is the Levi metric with respect to the Levi form $d \theta=$ $i h_{1 \overline{1}} \theta^{1} \wedge \theta^{\overline{1}}$ and $R_{1 \overline{1}}=W h_{1 \overline{1}}$. This expression is analogous to the gradient Kähler-Ricci soliton where the constraint $\varphi_{11}=0$ is parallel to the infinitesimal automorphism condition in that case (cf. page 93 in [CCGG+]). This justifies the term "pseudogradient" in our definition in consideration of the theory of gradient Ricci soliton.

(iii) Gradient Ricci solitons can be viewed as generalized Einstein manifolds in the weighted geometry sense and analogue theory was built for pseudo-gradient CR Yamabe solitons in weighted pseudohermitian geometry CKL. In particular, a pseudogradient CR Yamabe soliton has constant Bakry-Émery pseudohermitian Ricci curvature

$$
\operatorname{Ric}\left(\Delta_{\varphi}\right)(X, X):=R_{1 \overline{1}} X^{1} X^{\overline{1}}+\operatorname{Re}\left[\varphi_{1 \overline{1}} X^{1} X^{\overline{1}}\right]=\mu|X|^{2}
$$

and the Bakry-Émery pseudohermitian torsion of it is the same as the pseudohermitian torsion:

$$
\operatorname{Tor}\left(\Delta_{\varphi}\right)(X, X):=2 \operatorname{Re}\left[\left(i A_{\overline{1} \overline{1}}+\varphi_{\overline{1} \overline{1}}\right) X_{1} X_{1}\right]=\operatorname{Tor}(X, X),
$$

where $X=X^{1} Z_{1}+X^{\overline{1}} Z_{\overline{1}} \in T_{1,0} \oplus T_{0,1}$.

Similar to the case of complete gradient Ricci solitons, we have

Lemma 4.1. Let $\left(M^{3}, J, \theta, \varphi, \mu\right)$ be a complete three-dimensional pseudo-gradient CR Yamabe soliton of vanishing torsion, then

for some constant $C$;

$$
W+\frac{1}{2}\left|\nabla_{b} \varphi\right|^{2}-\mu \varphi=C
$$

(ii)

$$
\nabla_{b}\left(W e^{-\varphi}\right)=0 .
$$

Proof. (i) Since $0=\varphi_{0}=-i\left(\varphi_{1 \overline{1}}-\varphi_{\overline{1} 1}\right)$, we have

$$
\Delta_{b} \varphi=2 \varphi_{1 \overline{1}} \text {. }
$$

It follows from (4.1) that

$$
W_{1}+\varphi_{1 \overline{1} 1}=0
$$


By the commutation relation (2.4),$\varphi_{0}=0$ and $\varphi_{11}=0$,

$$
\begin{aligned}
\varphi_{1 \overline{1} 1} & =\varphi_{11 \overline{1}}-i \varphi_{10}-W \varphi_{1} \\
& =i \varphi_{01}+i A_{11} \varphi_{\overline{1}}-W \varphi_{1} \\
& =i A_{11} \varphi_{\overline{1}}-W \varphi_{1} .
\end{aligned}
$$

Hence, from (4.3) and (4.4), we know that

$$
W_{1}=-i A_{11} \varphi_{\overline{1}}+W \varphi_{1} .
$$

It follows from (4.5) and $\varphi_{11}=0$ that

$$
\begin{aligned}
\left(W+\frac{1}{2}\left|\nabla_{b} \varphi\right|^{2}-\mu \varphi\right)_{1} & =W_{1}+\left(\varphi_{1} \varphi_{\overline{1}}\right)_{1}-\mu \varphi_{1} \\
& =-i A_{11} \varphi_{\overline{1}}+(W-\mu) \varphi_{1}+\varphi_{1} \varphi_{\overline{1} 1} \\
& =-i A_{11} \varphi_{\overline{1}}-\varphi_{\overline{1} 1} \varphi_{1}+\varphi_{1} \varphi_{\overline{1} 1} \\
& =-i A_{11} \varphi_{\overline{1}} .
\end{aligned}
$$

Hence,

and

$$
\left(W+\frac{1}{2}\left|\nabla_{b} \varphi\right|^{2}-\mu \varphi\right)_{1}=0
$$

for some constant $C$ if $A_{11}=0$.

$$
\frac{1}{2}\left|\nabla_{b} \varphi\right|^{2}+W-\mu \varphi=C
$$

(ii) If $A_{11}=0$, then

$$
\begin{aligned}
\left(W e^{-\varphi}\right)_{1} & =\left(W_{1}-W \varphi_{1}\right) e^{-\varphi} \\
& =\left(-i A_{11} \varphi_{\overline{1}}+W \varphi_{1}-W \varphi_{1}\right) e^{-\varphi} \\
& =-i A_{11} \varphi_{\overline{1}} e^{-\varphi} \\
& =0
\end{aligned}
$$

We rewrite the real version of complete pseudo-gradient CR Yamabe soliton as follows:

Lemma 4.2. Let $\left(M^{3}, J, \theta\right)$ be a complete three-dimensional pseudohermitian manifold. Then $\left(M^{3}, J, \theta, \varphi, \mu\right)$ is a complete pseudo-gradient CR Yamabe soliton if and only if the following identities hold:

(ii)

$$
\varphi_{e_{1} e_{1}}=\varphi_{e_{2} e_{2}} \quad \text { and } \quad \varphi_{e_{1} e_{2}}=\varphi_{e_{2} e_{1}}=0
$$

$$
W+\frac{1}{2} \varphi_{e_{1} e_{1}}=W+\frac{1}{2} \varphi_{e_{2} e_{2}}=\mu .
$$

Proof. (i) From the definitions of $Z_{1}=\frac{1}{2}\left(e_{1}-i e_{2}\right)$ and $\varphi_{e_{i} e_{j}}=e_{j} e_{i} \varphi-\sigma_{i}^{k}\left(e_{j}\right) \varphi_{e_{k}}$, we have

and

$$
\varphi_{1}=\frac{1}{2}\left(\varphi_{e_{1}}-i \varphi_{e_{2}}\right)
$$

$$
\begin{aligned}
4 \varphi_{11}= & 4\left[Z_{1} \varphi_{1}-\theta_{1}^{1}\left(Z_{1}\right) \varphi_{1}\right] \\
= & 4\left[Z_{1} \varphi_{1}-i \sigma_{1}^{2}\left(Z_{1}\right) \varphi_{1}\right] \\
= & \left(e_{1} e_{1} \varphi-\sigma_{1}^{2}\left(e_{1}\right) \varphi_{e_{2}}\right)-\left(e_{2} e_{2} \varphi-\sigma_{2}^{1}\left(e_{2}\right) \varphi_{e_{1}}\right) \\
& -i\left(e_{1} e_{2} \varphi-\sigma_{2}^{1}\left(e_{1}\right) \varphi_{e_{1}}\right)-i\left(e_{2} e_{1} \varphi-\sigma_{1}^{2}\left(e_{2}\right) \varphi_{e_{2}}\right) \\
= & \left(\varphi_{e_{1} e_{1}}-\varphi_{e_{2} e_{2}}\right)-i\left(\varphi_{e_{1} e_{2}}+\varphi_{e_{2} e_{1}}\right) .
\end{aligned}
$$


On the other hand, from (2.7) we see that $\varphi_{0}=0$ if and only if

$$
\varphi_{e_{1} e_{2}}=\varphi_{e_{2} e_{1}} .
$$

Thus, we conclude that $\varphi_{11}=0$ and $\varphi_{0}=0$ if and only if

$$
\varphi_{e_{1} e_{1}}=\varphi_{e_{2} e_{2}} \quad \text { and } \quad \varphi_{e_{1} e_{2}}=\varphi_{e_{2} e_{1}}=0 \text {. }
$$

(ii) follows from Eq. (4.1) in the Definition 4.1, (2.6), and part (i) proved above.

\section{The Structure of Pseudo-Gradient CR Yamabe Solitons}

In this section, by using ideas of the recent work of the first-named author with $\mathrm{X}$. Sun and Y. Zhang [CSZ] on the structure of Yamabe gradient solitons, we obtain the structure of pseudo-gradient CR Yamabe solitons of vanishing torsion.

Let $\left\{\omega^{1}:=\operatorname{Re}\left(\theta^{1}\right), \omega^{2}:=\operatorname{Im}\left(\theta^{1}\right), \omega^{3}:=\lambda^{-1} \theta\right\}$ be the orthonormal coframe dual to $\left\{e_{1}, e_{2}, e_{3}=\lambda \mathbf{T}\right\}$ with respect to the Webster adapted metric $h^{\lambda}$ defined on $(M, J, \theta)$ as in Section 4. Then we have the following Riemannian structure equations:

$$
\begin{aligned}
d \omega^{\alpha} & =\omega^{\beta} \wedge \omega_{\beta}^{\alpha}, \quad 1 \leqslant \alpha, \beta \leqslant 3, \\
0 & =\omega_{\alpha}^{\beta}+\omega_{\beta}^{\alpha}, \\
d \omega_{\alpha}^{\beta} & =\omega_{\alpha}^{\gamma} \wedge \omega_{\gamma}^{\beta}+\frac{1}{2} R_{\alpha \beta \gamma \delta}^{\lambda} \omega^{\gamma} \wedge \omega^{\delta}, \quad 1 \leqslant \alpha, \beta, \gamma, \delta \leqslant 3,
\end{aligned}
$$

and $\theta^{1}=\omega^{1}+i \omega^{2}$ satisfies the structure equations as in (2.2) and (2.3). Here $R_{\alpha \beta \gamma \delta}^{\lambda}$ denotes the Riemannian curvature tensor.

Moreover, from [CChi, (3.6) and (3.7)] we have

$$
\theta_{1}^{1}=i\left(\omega_{1}^{2}-\lambda^{-2} \theta\right)
$$

and

$$
\begin{aligned}
\omega_{1}^{3} & =\left(-\lambda \operatorname{Re} A_{\overline{1} \overline{1}}\right) \omega^{1}+\left(-\lambda \operatorname{Im} A_{\overline{1} \overline{1}}+\lambda^{-1}\right) \omega^{2}, \\
\omega_{2}^{3} & =\left(-\lambda \operatorname{Im} A_{\overline{1} \overline{1}}-\lambda^{-1}\right) \omega^{1}+\left(\lambda \operatorname{Re} A_{\overline{1} \overline{1}}\right) \omega^{2} .
\end{aligned}
$$

Now we state the relation between the Ricci tensor $R_{i j}^{\lambda}$ of the Webster metric $h^{\lambda}$ and the pseudohermitian Ricci tensor $R_{1 \overline{1}}=W h_{1 \overline{1}}$.

Lemma 5.1. (CChi, Theorem 3.1]) Let $(M, J, \theta)$ be a complete pseudohermitian 3manifold and $R_{\alpha \beta}^{\lambda}$ be the Ricci curvature tensor with respect to the Webster metric $h^{\lambda}$ for some positive number $\lambda>0$. If the pseudohermitian torsion $A_{11}$ vanishes, then

$$
\left(R_{\alpha \beta}^{\lambda}\right)=\left(\begin{array}{ccc}
2 W-2 \lambda^{-2} & 0 & 0 \\
0 & 2 W-2 \lambda^{-2} & 0 \\
0 & 0 & 2 \lambda^{-2}
\end{array}\right) .
$$

Here $W$ is the Tanaka-Webster scalar curvature. In particular, the scalar curvature

$$
R^{\lambda}=4 W-2 \lambda^{-2} \text {. }
$$

Now we are ready to prove the classification theorem of complete pseudo-gradient CR Yamabe solitons.

Lemma 5.2. Let $\left(M^{3}, J, \theta, \varphi, \mu\right)$ be a complete pseudo-gradient CR Yamabe soliton. Then $\left|\nabla_{b} \varphi\right|$ is constant on each level set of the potential function $\varphi$. 
Proof. One computes that

$$
\begin{aligned}
\nabla\left(\left|\nabla_{b} \varphi\right|^{2}\right) & =\left(\varphi_{1} \varphi_{\overline{1}}\right)_{1} Z_{\overline{1}}+\left(\varphi_{1} \varphi_{\overline{1}}\right)_{\overline{1}} Z_{1} \\
& =(\mu-W) h_{\overline{1} 1} \varphi_{1} Z_{\overline{1}}+(\mu-W) h_{1 \overline{1}} \varphi_{\overline{1}} Z_{1} \\
& =(\mu-W) \nabla \varphi .
\end{aligned}
$$

So the level sets of $\left|\nabla_{b} \varphi\right|$ coincide with the level sets of $\varphi$.

Combining the lemma above with the soliton equation $W+\frac{1}{2} \Delta_{b} \varphi=\mu$ and the conserved quantity $W+\frac{1}{2}\left|\nabla_{b} \varphi\right|^{2}-\mu \varphi=C$, we immediately obtain the following

Lemma 5.3. Let $\left(M^{3}, J, \theta, \varphi, \mu\right)$ be a complete pseudo-gradient CR Yamabe soliton. Then, the potential function $\varphi$ is an isoparametric function on the Riemannain 3manifold $\left(M, h^{\lambda}\right)$.

Remark 5.1. In É. Cartan's study of Lie groups, a function $f$ is called an isoparametric function if both $|\nabla f|$ and $\Delta f$ depend only on the value of $f$ (i.e., constant on each level set of $f$ ). Note that, by the work of Q. M. Wang in [W] on isoparametric functions, the only possible singular level sets of the potential function $\varphi$ are smooth submanifolds

$$
\Sigma_{+}:=\left\{\varphi=\max _{x \in M} \varphi(x)\right\} \text { and } \Sigma_{-}:=\left\{\varphi=\min _{x \in M} \varphi(x)\right\},
$$

which are called the focal varieties of $\varphi$. Moreover, regular level sets are "tubes" over either $\Sigma_{+}$or $\Sigma_{-}$and these "tube" leaves together form foliations of tubular neighborhoods around either $\Sigma_{+}$or $\Sigma_{-}$. Finally, the argument in [W] can be further used to show that if an isoparametric function $f$ is locally constant then $f$ must be a constant function. Hence, the critical set of our non-trivial potential function $\varphi$ is at most 2dimensional. The relevant details will be provided in the Appendix for the reader's convenience.

\section{The Proof of Theorem 1.3 :}

Proof. In view of Lemma 5.3, it remains to classify the possible diffeomorphism types of $M^{3}$. Consider again the adapted metric

$$
g_{\lambda}:=\frac{1}{2} d \theta(\cdot, J \cdot)+\lambda^{-2} \theta^{2} .
$$

It follows from Lemma 5.1 that, when torsion vanishes,

$$
R i c^{\lambda}=\left(\begin{array}{ccc}
2 W-2 \lambda^{-2} & 0 & 0 \\
0 & 2 W-2 \lambda^{-2} & 0 \\
0 & 0 & 2 \lambda^{-2}
\end{array}\right)
$$

with respect to the orthonormal frame $\left\{e_{1}, e_{2}, e_{3}=\lambda \mathbf{T}\right\}$, where $e_{1}=Z_{1}+Z_{\overline{1}}$ and $e_{2}=i\left(Z_{1}-Z_{\overline{1}}\right)$.

When dimension $n=3$,

$$
R_{2323}^{\lambda}-R_{1313}^{\lambda}=R_{22}^{\lambda}-R_{11}^{\lambda}=0
$$

and

$$
R_{2323}^{\lambda}+R_{1313}^{\lambda}=R_{33}^{\lambda}=2 \lambda^{-2} .
$$

So we know that

$$
R_{2323}^{\lambda}=R_{1313}^{\lambda}=\lambda^{-2} \text {. }
$$


Moreover, let $V$ be any unit vector which is a linear combination of $e_{1}$ and $e_{2}$, then the sectional curvature of the two-plane spanned by $e_{3}$ and $V$ is merely a linear combination of $R_{2323}^{\lambda}$ and $R_{1313}^{\lambda}$, hence

$$
\operatorname{Rm}^{\lambda}\left(V, e_{3}, V, e_{3}\right)=\lambda^{-2}
$$

Note that this property holds only for the 3-dimensional case, because the Weyl tensor always vanishes and the curvature operator is diagonalized as long as $\operatorname{Ric}^{\lambda}$ and $g_{\lambda}$ are diagonalized.

Since by assumption the pseudo-gradient CR Yamabe soliton $M^{3}$ is non-trivial, $\varphi$ is not a constant function. By Lemma [5.2, if $\nabla \varphi \neq 0$ at some point $p$, then $\nabla \varphi \neq 0$ on the whole level set $\Sigma_{\varphi(p)}:=\{\varphi=\varphi(p)\}$. Such $\Sigma_{\varphi(p)}$ (with non-vanishing $\nabla \varphi$ ) are called regular. We are going to compute the Gaussian curvature of regular level surfaces.

Claim: Each regular level surface $\Sigma$ of $\varphi$ has zero Gaussian curvature.

Indeed, for any regular level surface $\Sigma$ of $\varphi$, first note that $\varphi_{0}=0$ means that $\mathbf{T}$ lies in the tangent subbundle $T \Sigma$ and is perpendicular to $\nabla \varphi$, so one can choose

$$
E_{1}=\frac{\nabla \varphi}{|\nabla \varphi|}=\alpha e_{1}+\beta e_{2} \text { and } E_{2}=\beta e_{1}-\alpha e_{2},
$$

where $\alpha, \beta$ are real-valued functions on $M$ such that $E_{1}, E_{2}$ and $E_{3}=e_{3}=\lambda \mathbf{T}$ form an orthonormal frame. The Gaussian curvature $K^{\lambda}$ of $\Sigma$ is given by the Gauss equation

$$
K^{\lambda}=R m^{\lambda}\left(E_{2}, E_{3}, E_{2}, E_{3}\right)-\operatorname{II}\left(E_{2}, E_{3}\right)^{2}+\operatorname{II}\left(E_{2}, E_{2}\right) \operatorname{II}\left(E_{3}, E_{3}\right),
$$

where II, the second fundamental form, can be computed via the connection 1-forms (cf. equation (5.3) )

$$
\omega_{1}^{3}=\lambda^{-1} \omega^{2} \text { and } \omega_{2}^{3}=-\lambda^{-1} \omega^{1}
$$

Indeed, we have

$$
\mathrm{II}\left(E_{3}, E_{3}\right)=\left\langle\nabla_{e_{3}} e_{3}, E_{1}\right\rangle=\omega_{3}^{1}\left(e_{3}\right)=0 .
$$

Similarly, since

$\nabla_{E_{2}} \mathbf{T}=\lambda^{-1} \nabla_{\beta e_{1}-\alpha e_{2}} e_{3}=\lambda^{-1}\left(\beta \omega_{3}^{k}\left(e_{1}\right) e_{k}-\alpha \omega_{3}^{k}\left(e_{2}\right) e_{k}\right)=\lambda^{-1}\left(\beta \lambda^{-1} e_{2}+\alpha \lambda^{-1} e_{1}\right)=\lambda^{-2} E_{1}$, we obtain

$$
\operatorname{II}\left(E_{2}, E_{3}\right)=\left\langle\nabla_{E_{2}} e_{3}, \frac{\nabla \varphi}{|\nabla \varphi|}\right\rangle=\lambda^{-1}
$$

Moreover, from the previous paragraph, we have known that $R m^{\lambda}\left(E_{2}, E_{3}, E_{2}, E_{3}\right)=$ $\lambda^{-2}$. Hence, $K^{\lambda}=0$.

So it follows that each regular level surface $\Sigma$ is isometric to either the flat torus $\mathbb{T}^{2}$, or the cylinder $\mathcal{C}=\mathbb{S}^{1} \times \mathbb{R}$, or the plane $\mathbb{R}^{2}$. Moreover, from the above computation, one sees that both $\nabla_{E_{3}} \mathbf{T}=0$ and $\nabla_{E_{2}} \mathbf{T}=0 \bmod E_{1}$, so $\mathbf{T}$ is parallel on the level surface with respect to the metric $g_{\Sigma}$ induced from $h^{\lambda}$. Note that the critical set of $\varphi$ cannot contain isolated points because $\varphi_{0}=0$ ensures that the trajectory along $\mathbf{T}$ starting from a critical point is necessarily contained in the critical set. Hence, each connected component of the critical set is at least 1-dimensional. On the other hand, by Lemma 6.2 , the potential function $\varphi$ cannot be locally constant so the critical set of $\varphi$ is at most 2-dimensional. Thus, the critical set of $\varphi$, if non-empty, may consist of only curves diffeomorphic to the real line or the circle, or surfaces diffeomorphic to the torus, the cylinder, or the plane (by Remark 5.1). Furthermore, if the critical set of $\varphi$ is non-empty it could contain a number of surfaces, but at most two curves because 
regular level surfaces shrinking to a critical curve will force the manifold to "close up". So we have the following three cases.

Case 1. The critical set of $\varphi$ contains no curves.

In this case, $M$ is diffeomorphic to either $\mathbb{R}^{3}, \mathbb{T}^{2} \times \mathbb{R}$, or $\mathbb{S}^{1} \times \mathbb{R}^{2}$ :

- When the critical set is empty, in view of Lemma 5.2 and by using the gradient flow of $\varphi$, it follows that $M$ is diffeomorphic to $\Sigma \times \mathbb{R}$, where $\Sigma=\mathbb{T}^{2}, \mathcal{C}$, or $\mathbb{R}^{2}$. This family of flat level surfaces is a foliation of $\left(M, h^{\lambda}\right)$.

- All critical components are 2-dimensional. If one of the critical surfaces is diffeomorphic to the torus $\mathbb{T}^{2}$, then by Remark 5.1 the regular level surfaces nearby are layers of the tubular neighborhood which move away from the critical $\mathbb{T}^{2}$ and form a foliation by tori until they encounter other critical surfaces. Note that other critical surfaces must be tori too. When such critical tori occur, there must be regular tori afterwards. So the regular foliation continues again. No matter how many critical tori are there, each is joined by foliations of regular tori. Thus, $M$ is diffeomorphic to $\mathbb{T}^{2} \times \mathbb{R}$. Similarly, if one of the ctitical surfaces is the cylinder $\mathcal{C}$, then all critical surfaces are cylinders and $M$ is diffeomorphic to $\mathcal{C} \times \mathbb{R} \approx S^{1} \times \mathbb{R}^{2} ;$ Finally, if one of the critical surfaces is $\mathbb{R}^{2}$, then all critical surfaces are $\mathbb{R}^{2}$ and $M$ is diffeomorphic to $\mathbb{R}^{3}$.

Case 2. The critical set of $\varphi$ contains only one curve $\gamma$.

In this case, $M$ is diffeomorphic to either $\mathbb{R}^{3}$ or $\mathbb{T}^{2} \times[0, \infty)$ with $\mathbb{T}^{2} \times\{0\}$ collapsing to $\mathbb{S}^{1}$ :

- When $\gamma$ is topologically the real line $\mathbb{R}$, nearby regular level surfaces must be the cylinder $\mathcal{C}$ which move away from $\gamma$ and become a foliation until a critical cylinder occurs. Similar to Case 1 , no matter how many successive critical cylinders are there, $M$ has to be diffeomorphic to $\mathbb{R}^{3}$ which is $\mathcal{C} \times[0, \infty)$ with $\mathcal{C} \times\{0\}$ collapsing to $\gamma$. The pseudo Gaussian solitons on the Heisenberg group with $\mu \neq 0$ are of this type and one can check that the adapted metric is

$$
h^{\lambda}=\left(e^{1}\right)^{2}+\left(e^{2}\right)^{2}+\lambda^{-2} \theta^{2}=\left(E^{1}\right)^{2}+\left(E^{2}\right)^{2}+\lambda^{-2} \theta^{2} .
$$

- When $\gamma$ is a closed curve (i.e., topologically a circle), regular level surfaces must be $\mathbb{T}^{2}$. Therefore, $M$ is diffeomorphic to $\mathbb{T}^{2} \times[0, \infty)$ with $\mathbb{T}^{2} \times\{0\}$ collapsing to $\mathbb{S}^{1}$. This can be realized by taking a quotient of the pseudo Gaussian solitons on the Heisenberg group such that the critical line becomes the critical circle.

Case 3. The critical set of $\varphi$ contains two curves $\gamma_{1}$ and $\gamma_{2}$.

In this case, $\mathrm{M}$ is diffeomorphic to either $\mathbb{S}^{2} \times \mathbb{R}^{1}, \mathbb{S}^{3}$, or the lens spaces $L(p, q)$ with $1 \leq q<p$ :

Since the regular level surfaces are tubes over either $\gamma_{1}$ or $\gamma_{2}$ and they form tubular neighborhoods of both $\gamma_{1}$ and $\gamma_{2}$, the two curves must both be lines or both be closed curves. As before, they are joined by either a family of cylinders or a family of tori, no matter whether these surfaces are critical or regular.

- When $\gamma_{1}$ and $\gamma_{2}$ are lines, regular level surfaces must be $\mathcal{C}$ and $\mathrm{M}$ is diffeomorphic to $\mathbb{S}^{2} \times \mathbb{R}^{1}$, where the $\gamma_{i}(i=1,2)$ correspond to $\{N\} \times \mathbb{R}^{1},\{S\} \times \mathbb{R}^{1}$ and cylinders are $K \times \mathbb{R}^{1}$. Here $N, S$ are the north and south poles respectively, and the $K$ 's are horizontal circles $\mathbb{S}^{2} \cap\{z=$ constant $c\}$ in $\mathbb{S}^{2}$. 
- When $\gamma_{1}$ and $\gamma_{2}$ are closed curves, $M$ admits a foliation of flat tori which degenerates at $\gamma_{1}$ and $\gamma_{2}$. In particular, $M$ admits a Heegaard splitting of two solid tori which means that $M$ is diffeomorphic to $\mathbb{S}^{3}$, or $\mathbb{S}^{2} \times \mathbb{S}^{1}$, or the lens spaces $L(p, q)$ with $1 \leq q<p$. However, $\mathbb{S}^{2} \times \mathbb{S}^{1}$ can be excluded because the first Betti number of a torsion free closed CR manifold must be even (cf. Appendix of [CH] or Theorem 4.3 in [T]).

To summarize, $M$ is diffeomorphic to one of the following spacess:

$\mathbb{R}^{3}, S^{3}, L(p, q), S^{2} \times \mathbb{R}, S^{1} \times \mathbb{R}^{2}, \mathbb{T}^{2} \times \mathbb{R}, \mathbb{T}^{2} \times[0, \infty)$ with $\mathbb{T}^{2} \times\{0\}$ collapsing to $S^{1}$.

\section{The Proof of Corollary 1.3 :}

Proof. We have mentioned in Remark 5.1 that the critical set of $\varphi$ consists of $\Sigma_{+}$ and $\Sigma_{-}$. When $\Delta \varphi=\Delta_{b} \varphi=2(\mu-W)$ does not change sign, $\varphi$ cannot attain both minimum and maximum on $M$ and thus $M$ is non-compact.

Suppose that $W>\mu$, then $\Delta \varphi<0$ and $\Sigma_{-}$must be empty. If $\Sigma_{+}$is also empty, then by Theorem 1.3 (i) $M$ must be diffeomorphic to $\mathbb{R}^{3}$ since it is simply connected. On ther other hand, if $\Sigma_{+}$is non-empty then it has to be connected for otherwise $\varphi$ must attain a local minimum somewhere, which is impossible. Hence the critical set of $\varphi$ has exactly one component. It follows from this fact, the assmption of $M$ being simply connected, and Theorem 1.3 (i) (ii) that $M$ is necessarily diffeomorphic to $\mathbb{R}^{3}$ (note that the critical set of $\varphi$ for $S^{2} \times \mathbb{R}$ consists of two lines, so it is not allowed here). Therefore, the only non-trivial simply-connected pseudo-gradient CR Yamabe soliton with $W>\mu$ must be diffeomorphic to $\mathbb{R}^{3}$.

The case $W<\mu$ can be proved similarly.

\section{Appendix}

We include some basic facts about isoparametric functions mentioned in Remark 5.1. Recall that our isoparametric potential function $\varphi$ satisfies $|\nabla \varphi|=b(\varphi)$ for some function $b: \mathcal{R} \rightarrow \mathbb{R}$, where $\mathcal{R}$ denotes the range of $\varphi$.

Lemma 6.1 (cf. Lemma 3 in [W]). The only possible singular level sets of $\varphi$ are the smooth focal submanifolds

$$
\Sigma_{+}:=\left\{\varphi=\max _{x \in M} \varphi(x)\right\} \text { and } \Sigma_{-}:=\left\{\varphi=\min _{x \in M} \varphi(x)\right\} .
$$

Proof. We follow the argument in [W]. Suppose that $b=|\nabla \varphi|=0$ on the level set $\Sigma_{c}:=\{x \in M \mid \varphi(x)=c\}$ for some critical value $c \in\left(\inf _{M} \varphi, \sup _{M} \varphi\right)$. Then, there exists some number $\epsilon>0$ sufficiently small so that $c$ is the only critical value in interval $[c, c+\epsilon]$. In particular, we have

$$
\int_{c}^{c+\epsilon} \frac{1}{|\nabla \varphi|} d \varphi=\lim _{a \rightarrow c^{+}} \int_{a}^{c+\epsilon} \frac{1}{|\nabla \varphi|} d \varphi=\epsilon .
$$

On the other hand, since $b \geq 0$ on $\mathcal{R}$ and $c$ is an interior point in $\mathcal{R}$, one has $b^{\prime}=0$ at $c$ so the function $b$ is locally bounded by its Taylor's reminder around $c$, i.e., $b(x) \leq A(x-c)^{2}$ for $x \in[c, c+\epsilon]$ and some constant $A>0$. However, this would imply that

$$
\int_{c}^{c+\epsilon} \frac{1}{|\nabla \varphi|} d \varphi \geq \int_{c}^{c+\epsilon} \frac{1}{A(x-c)^{2}} d \varphi=\infty
$$


which contradicts (6.1).

Suppose that $\varphi$ is locally a constant function on some open set $U$ of $M$, then $b$ and $b^{\prime}$ will vanish at the boundary of $U$, which is impossible as shown in the proof of Lemma 6.1. Therefore, we have the following lemma (which is used in the proof of Theorem 1.3).

Lemma 6.2. The non-constant potential function $\varphi$ cannot be locally constant. In particular, its critical set is at most 2-dimensional.

\section{REFERENCES}

[Br] L. E. J. Brouwer, Über Abbildung von Mannigfaltigkeiten, Math. Ann., 71 (1912), 97-115.

[Cao] H.-D. Cao, On Harnack's inequalities for the Kähler-Ricci flow, Invent. Math., 109 (1992), 247-263.

[CaoC] J. Cao and S.-C. Chang, Pseudo-Einstein and Q-flat metrics with eigenvalue estimates on CR-hypersurfaces, Indiana Univ. Math. J., 56 (2007), 2840-2857.

[CC] S.-C. Chang and J.-H. Cheng, The Harnack estimate for the Yamabe flow on CR manifolds of dimension 3, Ann. Glob. Anal. Geom., 21 (2002), 111-121.

[CCC] S.- C. Chang, J.- H. Cheng and H.-L. Chiu, A fourth order curvature flow on a CR 3-manifold, Indiana Univ. Math. J., 56 (2007), 1793-1825.

[CCGG+] B. Chow, S.-C. Chu, D. Glickenstein, C. Guenther, J. Isenberg, T. Ivey, D. Knopf, P. Lu, F. Luo and L. Ni, The Ricci flow: Techniques and Applications. Volume 2 - Part I: Geometric Aspects, Amer. Math. Soc., Providence, RI, 2007.

[CChi] S.-C. Chang and H.-L. Chiu, On the CR analogue of Obata's theorem in a pseudohermitian 3-Manifold, Math. Ann., 345 (2009), 33-51.

[CCW] S.-C. Chang, H.-L. Chiu and C.-T. Wu, The Li-Yau-Hamilton inequality for Yamabe flow on a closed CR 3-manifold, Trans. Amer. Math. Soc., 362 (2010), 1681-1698.

[CCZ] H.-D. Cao, B.-L. Chen and X.-P. Zhu, Recent Developments on Hamiltons Ricci flow Surv. Diff. Geom., Vol. XII (2008), 47-112.

[CH] S.S. Chern and R.S. Hamilton, On Riemannian metrics adapted to three-dimensional contact manifolds, Lecture Notes in Math., 1111, Springer, Berlin (1985), 279-308.

[Ch3] B. Chow, The Yamabe flow on locally conformally flat manifolds with positive Ricci curvature, Comm. Pure Appl. Math., 45 (1992), 1003-1014.

[Chen] B.-L. Chen, Strong uniqueness of the Ricci flow, J. Diff. Geom., 82 (2009), 363-382.

[Cho] W.-L. Chow, Über System Von Linearen Partiellen Differentialgleichungen erster Orduung, Math. Ann., 117 (1939), 98-105.

[CKL] S.-C. Chang, T.-J. Kuo and S.-H. Lai, CR Li-Yau gradient estimate and linear entropy formulae for Witten Laplacian via Bakry-Emery pseudohermitian Ricci curvature, submitted.

[CKW] S.-C. Chang, Otto van Koert and C.-T. Wu, The torsion flow on a closed pseudohermitian 3-manifold, preprint.

[CL] J.-H. Cheng and J. M. Lee, The Burns-Epstein invariant and deformation of the CR structures, Duke Math. J., 60 (1990), 221-254.

[CLN] B. Chow, P. Lu, L. Ni, Hamilton's Ricci flow. Grad. Stud. Math., 77. Amer. Math. Soc., Providende, RI, 2006.

[CMM] G. Catino, C. Mantegazza, and L. Mazzieri, On the global structure of conformal gradient solitons with nonnegative Ricci tensor, Commun. Contemp. Math. 14 1250045(2012), no. 6, 12 pages.

[CS] S.-C. Chang and T. Saotome, The Q-curvature flow in a closed CR 3-manifold, Proceedings of the 15th International Workshop on Differential Geometry and the 4th KNUGRG-OCAMI Differential Geometry Workshop [Volume 15], 57-69. Natl. Inst. Math. Sci. (NIMS), Taejŏn, 2011.

[CSZ] H.-D. Cao, X. Sun and Y. Zhang, On the structure of gradient Yamabe solitons, Math. Res. Lett., 19 (2012), 767-774. 
[DS] P. Daskalopoulos and N. Sesum, The classification of locally conformally flat Yamabe solitons, Adv. Math. 240 (2013), 346-369.

[DT] S. Dragomir and G. Tomassini, Differential Geometry and Analysis on CR manifolds, Progress in Mathematics, 246, Birkhäuser, Basel, 2006.

[FH] C. Fefferman and K. Hirachi, Ambient metric construction of $Q$-curvature in conformal and CR geometries, Math. Res. Lett., 10 (2003), 819-831.

[G] J. W. Gray, Some global properties of contact structures, Ann. Math.,69 (1959), 421-450.

[GL] C. R. Graham and J. M. Lee, Smooth solutions of degenerate Laplacians on strictly pseudoconvex domains, Duke Math. J., 57 (1988), 697-720.

[GY] N. Gamara and Y. Yacoub, CR Yamabe conjecture-the conformally flat case, Pacific J. Math., 201 (2001), 121-175.

[H1] R. S. Hamilton, Three-Manifolds with Positive Ricci Curvature, J. Diff. Geom., 17 (1982), 255-306.

[H2] R. S. Hamilton, The Ricci flow on surfaces, Mathematics and general relativity (Santa Cruz, CA, 1986), 237-262. Amer. Math. Soc., Providence, RI, 1988.

[H3] R. S. Hamilton, The Harnack estimate for the Ricci flow, J. Diff. Geom., 37 (1993), 225-243.

[H4] R. S. Hamilton, A matrix Harnack estimate for the heat equation, Comm. Anal. Geom., 1 (1993), 113-126.

[H5] R. S. Hamilton, The formation of singularities in the Ricci flow, Surveys in differential geometry, Vol. II (Cambridge, MA, 1993), 7-136. Internat. Press, Cambridge, MA, 1995.

[H6] R. S. Hamilton, Lectures on geometric flows, 1989, unpublished.

[Hi] K. Hirachi, Scalar pseudo-hermitian invariants and the Szegö Kernel on 3-dimensional CR Manifolds, Lecture Notes in Pure and Appl. Math. 143, 67-76. Dekker, 1992.

[Ho] P. T. Ho, The long time existence and convergence of the CR Yamabe flow, Commun. Contemp. Math. 14 (2012), 50 p.

[Hsu] S.-Y. Hsu, A note on compact gradient Yamabe solitons, J. Math. Anal. Appli., 388 (2012), $725-726$.

[I] T. Ivy, Local existence of Ricci solitons, Manuscripta Math., 91 (1996), 151-162.

[KT] Y. Kamishima and T. Tsuboi, CR-structures on Seifert manifolds, Invent. Math., 104 (1991), 149-163.

[L1] J. M. Lee, Pseudo-Einstein structure on CR Manifolds, Amer. J. Math., 110 (1988), 157-178.

[L2] J. M. Lee, The Fefferman metric and pseudohermitian invariants, Trans. Amer. Math. Soc., 296 (1986), 411-429.

[P1] G. Perelman, The entropy formula for the Ricci flow and its geometric applications, arXiv:math.DG/0211159.

[P2] G. Perelman, Ricci flow with surgery on three manifolds, arXiv:math.DG/0303109.

[Ru] M. Rumin, Formes Différentielles sur les variétés de contact, J. Diff. Geom., 39 (1994), 281-330.

[SS] H. Schwetlick and M. Struwe, Convergence of the Yamabe flow for large energies, J. Reine Angew. Math., 562 (2003), 59-100.

[T] S. Tachibana, On harmonic tensors in compact Sasakian spaces, Tohoku Math. J., 17 (1965), 271-284.

[W] Q.-M. Wang, Isoparametric functions on Riemannian manifolds. I, Math. Ann., 277 (1987), 639-646.

${ }^{1}$ Department of Mathematics, University of Macau, Macao \& Lehigh University, BethleheM, PA 18015, USA

E-mail address: huc2@lehigh.edu

${ }^{2}$ Department of Mathematics and Taida Institute for Mathematical Sciences (TIMS), National Taiwan University, Taipei 10617, Taiwan, R.O.C.

E-mail address: scchang@math.ntu.edu.tw

${ }^{3}$ Department of Mathematics, National Taiwan University, Taipei 10617, Taiwan, R.O.C.

E-mail address: BabbageTW@gmail.com 